\title{
The Role of Maps in Autonomous Driving Simulations
}

\author{
Árpád Barsi1, András Csepinszky², János Máté Lógó1, Nikol Krausz¹, Vivien Potó1 \\ ${ }^{1}$ Department of Photogrammetry and Geoinformatics, Faculty of Civil Engineering, Budapest University of Technology and \\ Economics, H-1111 Budapest, Műegyetem rkp. 3., Hungary \\ 2 NNG Global Infotainment Technologies, H-1037 Budapest, Szépvölgyi út 35-37., Hungary \\ * Corresponding author, e-mail: barsi.arpad@epito.bme.hu
}

Received: 03 March 2020, Accepted: 11 March 2020, Published online: 13 August 2020

\begin{abstract}
The vehicles of the conditional, high, and full automation levels have a common unique sensor, the map. The term map has undergone a significant change because the spatial resolution has been increased considerably, the road infrastructure and its neighborhood are represented with higher accuracy in 3D. The development of these vehicles requires enormous efforts, where computer-based techniques, like the simulations, can offer a helping hand. The autonomous simulations will be supported by high-quality map information, which generates interest in the best field data-capturing techniques. The paper provides an overview of the available modern surveying methodologies, then introduces the most preferred data formats - both in physical information storage and in exchanging information content between mapping systems. Some examples are presented to demonstrate the usage of the relevant map-making outputs in automotive simulators.
\end{abstract}

Keywords

high-definition map, environmental modeling, mapping technology

\section{Introduction}

"The fully automated vehicle drives by itself without human supervision" - this definition has been taken from the German Federal Highway Research Institute (BASt) (Gasser et al., 2012:p.14). Prior to achieving this ideal situation, several milestones must be reached, which are intermediate stages in the development. The evolution of technology might be characterized by integrated computer services, which have three main categories:

- Driver Information Systems (DIS): the main goal is to support the driver by information about the vehicle's status, environment, planned route, etc.

- Driver Assistance Systems (DAS): the aim to help in the driving in controlling, e.g., the speed, brake system,

- Advanced Driver Assistance Systems (ADAS): the purpose is to establish a higher level service in the drivers' support; e.g., complex control mechanism belongs to this group, like lane changing assistant, highway pilot, etc.

The above-characterized services are responsible for control, safety, or comfort. Another possible categorization is feasible after the relationship to geographic or map information. The most used and known DIS tool is the navigation, where the service displays instructions to the driver helping him in reaching the desired destination, while the offered route fulfills the prior conditions (length, cost efficiency, allowed road categories, etc.). A typical ADAS technique with strong map support is illustrated by the curve warning or wrong-way (ghost driving) warning. A more developed assistant system is an example of an intersection collision avoidance, hill descent control, or fuel-efficiency advisor (Winner et al., 2016).

It is trivial that the well-defined SAE levels of autonomous driving require higher and higher support of computer hardware, software, and data (SAE International, 2014). To cope with the aimed level, an increased amount of development and tests are requested. The computer can nevertheless be involved also in this development procedure. The simulation has landed into the hottest focus. The real simulation environment cannot be imagined in another way, just with the integration of field survey and mapping technologies, coupled with the ability of simulator file output.

The paper organized as follows: Section 2 presents the map production technologies, which are nowadays available in the field survey practice and are suitable to offer relevant 
volume and quality of the requested data. In Section 3, the most important map data formats are reviewed, Section 4 gives an overview of the automotive simulation support from the mapping science. Finally, a Conclusion (Section 5) and References sections can be found.

\section{Map production technologies}

The traditional navigation maps are created for human use, and therefore the information content must be harmonized to the human-environmental perception. Road geometry and topology has been visualized in a simple way since the driver can spend just a limited time to read and interpret the stored information. The primary goal is to show the connections, and interesting objects, like buildings, Point of Interests (POIs), etc.

In contrast, the autonomous world has the computer to take over these tasks. Thanks to the high speed of data processing of the onboard computers, the data request has also been increased (Kleine-Besten et al., 2015). This reason has led to the development of the maps with respect to the resolution (Craig, 2012).

The human purposed maps are called Small Definition (SD) maps, while the more advanced and increased data density maps are the High Definition (HD) maps. All of the data acquisition techniques must be in synchronization to the later, the HD mapping purposes (HERE, 2019). The section presents so the potential "high throughput" map production technologies.

The satellite imagery is an excellent data source in remote sensing when the mapping area has a large extension, the accuracy is medium and homogenous, and the production efforts are small. Several satellite platforms offer a ground resolution of meter to submeter level, as Sentinel-2 MSI (MultiSpectral Instrument) 10-60 m, SPOT $1.5 \mathrm{~m}$, but Ikonos serves with $0.82 \mathrm{~m}$, QuickBird with $0.65 \mathrm{~m}$, GeoEye with $0.34 \mathrm{~m}$ or WorldView with $0.31 \mathrm{~m}$. Multispectral imaging means that the sensor is capable of capturing images in multiple spectral bands, typically 4 to 10 channels, and this makes the object recognition feasible mostly in an automatic way. The obtained recognition accuracy is 80 to $90 \%$ on average. As a disadvantage, one must mention the strong dependency on cloud coverage, additionally shadows and uncovers decrease the mapping quality.

Aerial mapping is based on photographs taken from airplanes, helicopters, or balloons. The science and technology of photogrammetry has a goal to generate homogenous high accuracy maps with possibly minimal fieldwork. As far as the airplanes can carry not only extreme precise cameras but also Lidars, the data capture is extended by aerial laser scanning. Airborne laser scanning offers detailed digital surface models, which are crucial in deriving digital orthophotos. An orthophoto is a product, which has similar features like the raw image, like color tone (the so-called intensity) values, but isn't distorted by the perspective and height effects. (An orthophoto can be taken as an exactly vertical projected aerial image, where all field objects can be drawn around, and the so obtained polygons have no distortions). These products are more informative than traditional maps, where only interesting and prior selected objects are evaluated and mapped by simple lines.

Aerial mapping technology is nowadays understood also with the use of Unmanned Aerial Systems (UAS), too. The drone technology is very flexible concerning the flying height, imaging density, or image orientation but is sharply limited by factors as operating battery or platform payload capacity. Locally operated UAS can serve with very cheap data capturing mode and easy usage. Aerial mapping technologies are similarly depending on the weather circumstances, shadows and uncovers can still occur. Homogeneity and scalable resolution, efficiency for the even larger area are the advantages. Because the methods achieve images with color values, not only geometric elements can be mapped (e.g., road "ribbons" or their axes), but it has also the capability to extract descriptive information also on the state of the road, their material, and other similarly visible attributes. The geometric resolution of photogrammetry reaches the $\mathrm{cm}-\mathrm{dm}$ range with appropriate accuracy and reliability measures.

The field surveying is an ancient technology to establish highly accurate maps. Surveyors draw maps by evaluating the objects in the field after some distances and angles measured. Nowadays, the satellite-based technology of Global Positioning System (GPS) and further analogous methodologies, called Global Navigation Satellite System (GNSS), is also part of surveying discipline. These are the most precise but slowest methods in data acquisition. Apart from the low efficiency, this costly technology is not suitable for HD mapping. Resolution and geometric accuracy is about $\mathrm{cm}$ to $\mathrm{mm}$ order.

A distinct method in the field survey is the Terrestrial Laser Scanning (TLS), which uses a fix position Lidar instrument capturing a point cloud around it (Fig. 1). The scanner is mostly equipped by a color camera, which allows to color all the captured points. The obtained point cloud has a high spatial density and simultaneously accuracy, but requires enormous storage capacity and data 


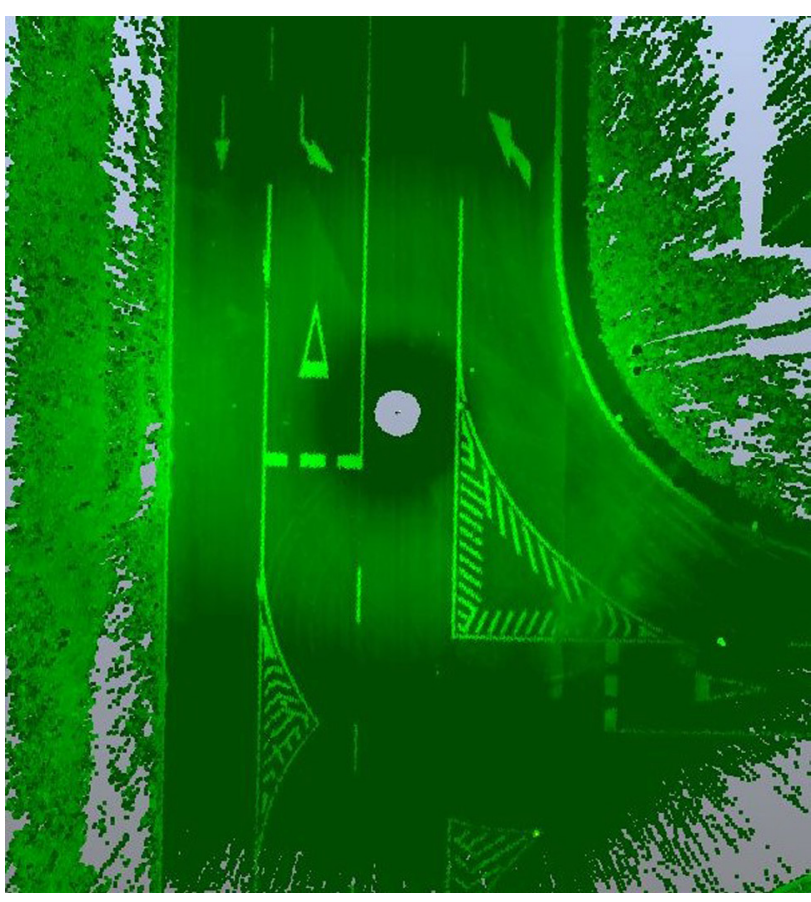

Fig. 1 Vertical view of a TLS point about a road intersection in ZalaZone

processing performance. Unintended shadows occur, which effects can be reduced by multiple-station methodology during the data capture. TLS also depends on weather conditions, although if purely the geometry is the goal, it can also be involved during nights thanks to its active remote sensing technology and energy source. Resolution reaches the $\mathrm{mm}$ range, while absolute accuracy is about $\mathrm{cm}$ to dm level. (Absolute accuracy is per definition on the whole Earth, while relative accuracy is meant just locally considering the neighboring points.)

The more mobile, so more efficient version of the Lidar data acquisition is the Mobile Laser Scanning (MLS). Since these solutions are mainly equipped not only by positioning systems (with GNSS, Inertial Measurement Units and odometers), but also cameras or camera systems, the name is frequently used as mobile mapping (Fig. 2). There is a general agreement that this is the most effective (concerning the data capturing capability, the obtained accuracy, and the costs), which can be involved in support of HD mapping. There is no doubt that mobile mapping results in much higher data amount, so the storage and processing has significantly more challenge. The suitability for deriving the best autonomous support is evident, considering the spatial data resolution and 3D-accuracy. The very expensive instrumentation is maybe the most severe disadvantage of technology.

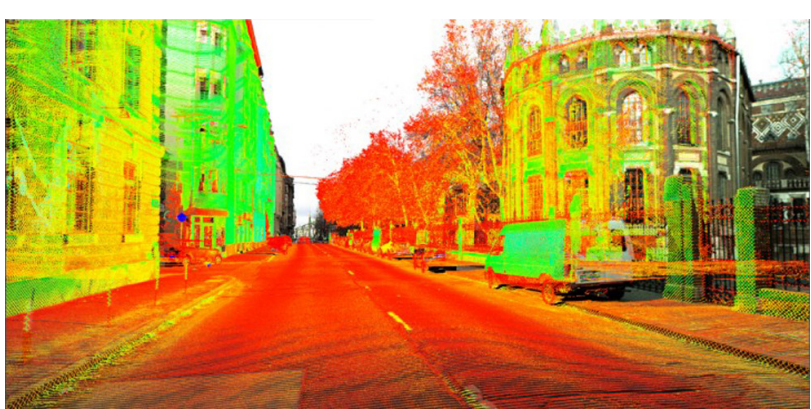

Fig. 2 A Lidar point cloud captured by a Leica Pegasus mobile mapping system

\section{Map data formats}

The captured massive amount of data has to be processed, and after the evaluation, the information has to be stored in one of the sophisticated map data formats. These formats have a great variety because of the different data processing chains and company traditions. There are two main groups for map data formats:

- physical data formats, which are primarily to store and use the information in the maps, and

- exchange data formats, which enables a gateway between the physical formats.

The physical map data formats can furthermore store the information content in binary or text (in ASCII) form. The latter is XML (Extensible Markup Language) or JSON (JavaScript Object Notation) in most cases. The current paper doesn't allow to present all relevant formats, but two significant examples can be summarized.

The first use case is the Navigation Data Standard (NDS), which is a format developed by a consortium of carmakers, mapping companies, research institutions, and universities (Behrens et al., 2015; Navigation Data Standard, 2018; Open Lane Model, 2019). The standard has been developed for size-efficient storage with solid performance requests on embedded devices. It uses SQLite as a file format and database engine. The data is stored in relational database tables with Binary Large Objects (BLOB) columns and is divided into building blocks as logical components; however, geographical partitioning by tiles is simultaneously applied. Its formal data description language is called Relational DataScript. NDS Association has decided to make RDS available to the public under the name zserio and continue its development (zserio, 2020). Each building block addresses specific functional aspects; there are blocks among others for basic map display, routing, natural guidance, lane, 
points of interest, traffic information. Additional blocks are to extend the indexing and advanced visualization techniques as junction view, orthoimages, and 3D objects.

The routing building block functionally supports the route calculation, route guidance, and also ADAS. It is based on the road level model with road geometry lines, intersections, and links. Transitions and turns are also modeled. The lane building block is responsible for lane geometry, lane boundaries and physical barriers, and road markings. It gives information on lane-changing possibilities, and lane properties, like directions, numberings, groups, and relations between lanes. It has a modeling tool for intersections and lane connectivity.

The second physical format example is the OpenDRIVE. OpenDRIVE is a member of the Open Standards family, which was developed to cover the macroscopic road geometry (OpenDRIVE), microscopic (pavement surface) geometry (OpenCRG), and traffic flow (OpenSCENARIO). OpenDRIVE was originally developed by Daimler for the analytical description of roads in a scalable way to support different use cases. It was the first open format to prescribe logical specifications and a 3D environment. Five additional companies joined to increase the standard's usability, among them Vires and the German Aerospace Center (DLR). Now the Chinese company Baidu has created a dialect and used the standard in their onboard devices.

OpenDRIVE uses XML to store the road network geometry and its features. It is organized in nodes, having a strict syntax defined by the standard, although it also gives the possibility to the users to extend by their own defined data content. The main nodes are the header, road, junction, junction group, station, and controller. Under the road nodes the horizontal and vertical information, as well as the lane information, are stored. Road objects, infrastructure elements are also managed (Fig. 3).

OpenDRIVE is based on high accuracy field measurements; therefore, it has become a worldwide used standard, which is understood by many automotive simulations and computer analysis software.

The exchange map data formats are developed to transform the information content of the different physical map data formats into other physical formats without losses. It explains why the exchange formats are significantly complicated than physical ones.

The most frequently used exchange format is the Geographic Data File (GDF), which has the version 5.1

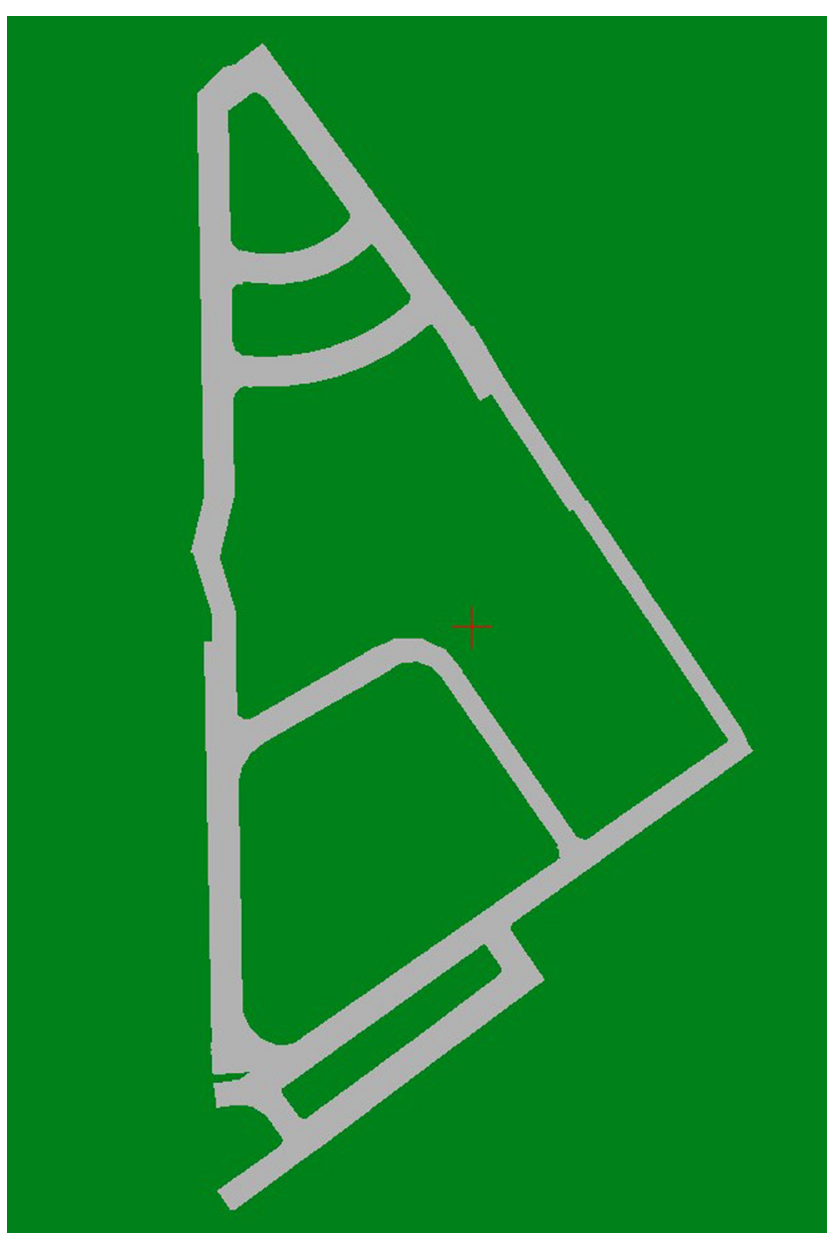

Fig. 3 OpenDRIVE model of a part of the BME campus

actually (European Committee for Standardization, 2018; International Organization for Standardization/Proof of a new International Standard, 2020; Wikipedia, 2020). This format was elaborated with respect to the geographic databases in Intelligent Transportation System applications and specified the conceptual and logical data model and physical encoding formats. The specification considers the potential contents of such databases (i.e., dictionaries for features, attributes, and relationships). These catalogs extended by unique element identifiers provide a mean of element identification regardless of a particular physical representation. The models consider the atomic built-up of the realizations; the four primitives are the points, lines, areas, and complex atoms. It manages the topology at an advanced, consistent level (with planar and non-planar mode). An interesting feature of GDF is the handling of abstraction levels with topological primitives (nodes, edges, etc.), simple and aggregated complex features. Hierarchic attribute modeling, relationships, height, and time management are also features of this standard. 


\section{Automotive simulation support}

The development of a vehicle and all of its parts requires a certain amount of testing. Because the physical tests of a prototype costs huge money and the fixing of the recognized errors and problems need time, the computer simulation had become a very crucial tool.

"A simulation is an approximate imitation of the operation of a process or system, that represents its operation over time." (Wikipedia, 2019). There is an alternative definition as "Simulation is the implementation of a model or models in a specific environment that allows the models' execution or use over time." (Walden et al., 2015).

The automotive development can profit from these technologies; namely, performance optimization can be executed, safety issues are easy to be studied and analyzed, different testings and validations can be realized, as well as training and education are much lighter than before. Simulation is an integral part of the production chain, started from the proof of concept validation, analysis, and increase of the complexity of the whole system (also its architecture, too). Simulation is enabling the rise in the level of quality (meant for the products and services). Error detection, failure localization, performance, and effectivity increase are further areas where simulation is generally beneficial. Simulation is better in cost aspects, too.

The vehicles are built of more and more complex parts; many of them contain microcontrollers or computers. The hardware and software ecosystem is an excellent arena for simulation. The vehicle as a whole or software elements (e.g., the ADAS assistants) might by tested in quasi-real circumstances if the simulator is equipped by the "functional" or "behavioral" copy of the reality. This copy implies the map information nevertheless.

The simulator packages shall, therefore, be supported by the map data formats (described in Section 3), so the field measurements are transferred into the simulators' virtual environment. Having the transformation proceed, the simulation scenario is created, where some sensors and control system components are to be added, and the experiments are ready to run. Simulations obtain excellent visualizations, numeric measures, or diagrams. The precise scenario can illustrate the analyzed vehicle movement comparable to the road infrastructure elements and the traffic (Fig. 4). It is also advantageous that scalable computing power is available, so the complex and numerically intensive analyses are available in a reduced time frame.

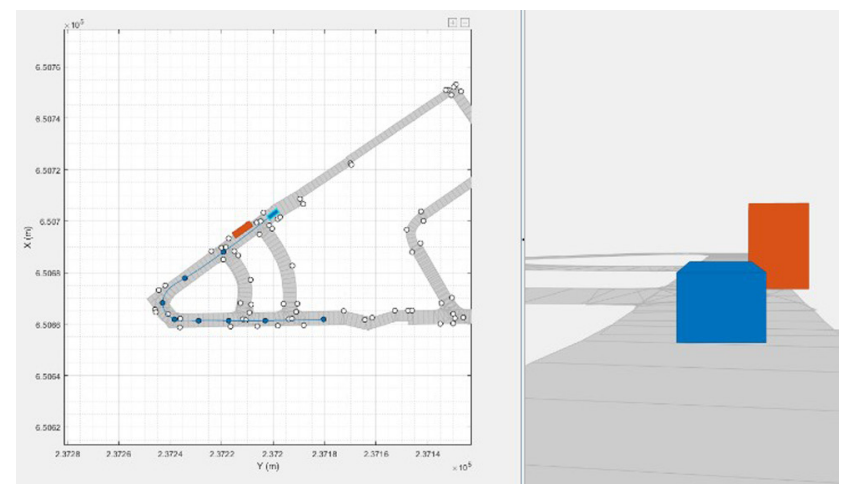

Fig. 4 Test case in Matlab Driving Scenario Designer with a car and truck. The base map is of the BME campus

\section{Conclusion}

The steady demand against information on the vehicles' environment was served by maps. The current map representation has a double task:

- it has to help the people to show the Earth surface and the main roads helping in goal setting during the navigation and

- it has to support the assistants and lastly the control of the vehicle, which is a machine type usage.

Although the first purpose has a long tradition, and this is not a cartographic paper, we do want to focus on the second aspect. The map can only help in the outlined struggle if it contains the necessary information in a proper format. The needed information is compiled during the field data capturing and processing work chain, whereas the format means (digital) databases with suitable data interfaces. The field surveying methods are nowadays capable of grabbing all fine details of the environment, which are and might be relevant in the vehicular usage. Acquiring camera imagery and Lidar spatial point cloud data sets forms the base; on top, the map-making technology can be built for autonomous applications. These inputs have the necessary resolution, accuracy, and object representation "power", which enables to transfer the information content into standardized data formats. These formats have been specified by a harmonized work of automotive and mapping experts, so they guarantee the ease and usability in the vehicular world. Especially automotive simulations can promptly profit from the available environmental representation. The technology development seems to struggle more efficiency, speed, and more prominent geographical coverage, including an increased variety of potential situations for better testing. 


\section{Acknowledgement}

The research reported in this paper was supported by the Higher Education Excellence Program in the frame of Artificial Intelligence research area of Budapest

\section{References}

Behrens, R., Kleine-Besten, T., Pöchmüller, W., Engelsberg, A. (2015) "Digitale Karten im Navigation Data Standard Format" (Digital Maps in Navigation Data Standard Format), In: Winner, H., Hakuli, S., Lotz, F., Singer, C. (eds.) Handbuch Fahrerassistenzsysteme, Springer Vieweg, Wiesbaden, Germany, pp. 513-523. (in German)

https://doi.org/10.1007/978-3-658-05734-3_27

Craig, J. (2012) "Map Data for ADAS", In: Eskandarian, A. (ed.) Handbook of Intelligent Vehicles, Springer, London, UK, pp. 881-892. https://doi.org/10.1007/978-0-85729-085-4_33

European Committee for Standardization (2018) "CEN/TS 17268:2018 Intelligent transport systems - ITS spatial data - Data exchange on changesinroadattributes",EuropeanCommitteeforStandardization, Brussels, Belgium. [online] Available at: https://standards.cen. $\mathrm{eu} / \mathrm{dyn} / \mathrm{www} / \mathrm{f}$ ?p=204:110:0::::FSP_PROJECT,FSP_ORG_ID: 65889,6259\&cs=1A219A77F5BD6DAC3FC5DD929FAE7AE97 [Accessed: 08 February 2020]

Gasser, T. M., Arzt, C., Ayoubi, M., Bartels, A., Bürkle, L., Eier, J., Flemisch, F., Häcker, D., Hesse, T., Huber, W., Lotz, C., Maurer, M., Ruth-Schumacher, S., Schwarz, J., Vogt, W. (2012) "Rechtsfolgen zunehmender Fahrzeugautomatisierung Fahrzeugtechnik: Gemeinsamer Schlussbericht der Projektgruppe" (Legal consequences of increasing vehicle automation, Vehicle technology: Joint final report of the project group), Wirtschaftsverlag NW Verlag für neue Wissenschaft $\mathrm{GmbH}$, Bergisch Gladbach, Germany. (in German)

HERE "HD Maps for Autonomous Driving and Driver Assistance | here", [online] Available at: https://www.here.com/products/automotive/ hd-maps [Accessed: 08 December 2019]

International Organization for Standardization/Proof of a new International Standard (2020) "ISO/PRF 20524-2 Intelligent transport systems - Geographic Data Files (GDF) - GDF5.1 - Part 2: Map data used in automated driving systems, Cooperative ITS, and multimodal transport", International Organization for Standardization, Geneva, Switzerland. [online] Available at: https://www.iso.org/ standard/72494.html [Accessed: 08 December 2019]
University of Technology and Economics (BME FIKP-MI/FM). The project has been supported by the European Union, co-financed by the European Social Fund. EFOP-3.6.3-VEKOP-16-2017-00001.

Kleine-Besten, T., Behrens, R., Pöchmüller, W., Engelsberg, A. (2015) "Digital maps for ADAS", In: Winner, H., Hakuli, S., Lotz, F., Singer, C. (eds.) Handbook of Driver Assistance Systems, Springer, Cham, Switzerland, pp. 647-661. https://doi.org/10.1007/978-3-319-12352-3_27

Navigation Data Standard "The worldwide standard for map data in automotive eco-systems", [online] Available at: https://www. nds-association.org/ [Accessed: 29 October 2018]

Open Lane Model "Open Lane Model: Navigation Data Standard", [online] Available at: http://www.openlanemodel.org/ [Accessed: 31 January 2019]

SAE International (2014) "Surface Vehicle Recommended Practice J3016 - Taxonomy and Definitions for Terms Related to Driving Automation Systems for On-Road Motor Vehicles", SAE International, Warrendale, PA, USA.

Walden, D. D., Roedler, G. J., Forsberg, K., Hamelin, R. D., Shortell, T. M. (2015) "Systems engineering handbook: a guide for system life cycle processes and activities", International Council on Systems Engineering, Hoboken, New Jersey, United States of America.

Wikipedia (2019) "Geographic Data Files", [online] Available at: https://en.wikipedia.org/wiki/Geographic_Data_Files [Accessed: 31 January 2020]

Wikipedia (2020) "Simulation", [online] Available at: https://en.wikipedia. org/wiki/Simulation [Accessed: 31 January 2020]

Winner, H., Hakuli, S., Lotz, F., Singer, C. (2016) "Handbook of Driver Assistance Systems: Basic Information, Components and Systems for Active Safety and Comfort", Springer, Cham, Switzerland. https://doi.org/10.1007/978-3-319-12352-3

zserio "zserio: Format Definition Tools", [online] Available at: http://zserio.org/ [Accessed: 08 February 2020] 